\title{
Time errors in a frequency controller
}

\section{Richard G. Brown ${ }^{1} \quad$ Gerrard Liddell ${ }^{2} \quad$ Nabil Adam $^{3}$}

(Received 11 September 2016; revised 8 March 2017)

\begin{abstract}
The problem provided by Transpower for the Mathematics in Industry New Zealand study group in 2015 was to identify the cause of ramping time errors in the electrical generation network that started appearing after the commissioning of the Frequency Keeping Controller on the High Voltage Direct Current link between the North and South islands. Three aspects of the problem were considered by subgroups of the main working group. First was an examination of the time error data, second was construction of simple dynamical models of the Frequency Keeping Controller, Multiple Frequency Keeping, and governor systems, and thirdly was an exploration of the Simulink control system that was provided by Transpower. The simplified model is presented in depth in this article. While the problem was not solved at the meeting, potential sources of the time error were identified, but a useful outcome from the meeting was the development of a reduced model of the power grid that can exhibit some of behaviour of the system.
\end{abstract}

DOI:10.21914/anziamj.v57i0.11308, (c) Austral. Mathematical Soc. 2017. Published April 12, 2017, as part of the Proceedings of the 2015 Mathematics and Statistics in Industry NZ Study Group. ISSN 1445-8810. (Print two pages per sheet of paper.) Copies of this article must not be made otherwise available on the internet; instead link directly to the DOI for this article. Record comments on this article via http://journal austms .org. au/ojs/index.php/ANZIAMJ/comment/add/11308/0 


\section{Contents}

1 Introduction

M269

2 Modelling whole-network frequency regulation

M272

2.1 Governor model-regulating governors . . . . . . . . . . M272

2.2 Island model . . . . . . . . . . . . . . . . . . . M273

2.3 MFK: island-level frequency control . . . . . . . . . . . M274

2.3.1 Some simple numerical experiments . . . . . . . M276

2.4 FKC: interisland frequency synchronisation . . . . . . . . M279

2.4.1 Some numerical experiments . . . . . . . . . . . M281

3 Conclusions, and future work

M281

\section{Introduction}

The New Zealand power grid has an unusual design, in that it has two separate island networks, North and South, whose AC frequencies are kept tightly synchronised via a high voltage direct current (HVDC) link between the islands. The power grid needs to supply power of constant frequency $(50 \mathrm{~Hz})$ and voltage to meet fluctuating loads with a mixture of hydroelectric, thermal, and wind sources.

Transpower is responsible for managing the network infrastructure, and regulating the power supply. As well as precisely regulating the $50 \mathrm{~Hz}$ frequency of the mains electricity system, Transpower are also responsible for regulating the time error, that is the error that would accumulate running a synchronous clock from the mains signal.

Each island essentially comprises a network of synchronous generators, each one of which is given a target amount of power generation at five minute intervals. This prescribed amount of generation is known as dispatch, and is optimised to meet expected demand for that that period. 
Frequency is controlled on each island by a Multiple Frequency Keeping (MFK) controller, which adjusts the set point generation values of a subset of participating generators in response to deviations in frequency from the nominal $50 \mathrm{~Hz}$. Additionally, a Frequency Keeping Controller (FKC) was commissioned in 2014 which enables deviations to be made to electrical current between islands through the High Voltage Direct Current (HVDC) link. The primary purpose of the link is to balance the asymmetry between load and generation between the islands, the FKC provides perturbations to this current. These corrections are made in response to frequency differences between the island, and the purpose of the FKC is to tightly couple the frequencies between islands, so that they behave as a single synchronised system.

Before the FKC controller was turned on, the time error on each island was variable, with occasional manual corrections from the Transpower operators being required to keep it within acceptable bounds. However, since the FKC controller has been turned on, the time error has been less variable, but, concerningly, has had a tendency to accumulate large errors rapidly. These errors are currently corrected by manual operator intervention.

The problem presented to the Mathematics in Industry New Zealand (MINZ) study group was to try to discover what properties in the complex control system network are the root cause of this variability in order to enable Transpower to better understand their system and to focus efforts in correcting this problem. Transpower provided significant amounts of data on frequency and load at fine resolution over a long time period, and also schematics of the various control systems involved.

As the problem was very complex, involving a very large power system network, with multiple controllers acting at different geographical and time scales, there was no one clear preferred approach to the problem. The first two days of the workshop were spent understanding the many interacting aspects of the problem, with Nabil Adam from Transpower providing patient explanations and teaching the participants about power and control systems.

The group then split into subgroups, according to expertise. There was a 
wealth of data to analyze, and a statistical understanding of this data was the focus of one group. Geoffrey Pritchard found that the behaviour of the time error resembles a random walk: a completely uncontrolled process whose fluctuations are driven only by random noise. The lag-one differences of the five-minute time error series are approximately normally distributed with zero mean, though with heavier tails, and successive differences are correlated with a correlation coefficient of 0.65 . When the random-walk idea is tested by constructing a process with independent increments re-sampled from those differences, that process quickly grows to typical amplitudes several times greater than observed in the time error. This suggests that, through some combination of the control systems and operator intervention, the time error is weakly mean-reverting.

Using a combination of regression and dynamic programming of shortest path methods, it has subsequently been possible to give a more systematic description of the this behaviour during the 202 state periods when the FKC was in regular operation. Details of this description will be the subject of a future publication.

Another group worked on constructing simple dynamical models of the FKC, MFK and governor system. This was capable of generating some of the observed behavior, such as an accumulating time error in some asymmetrical situations between islands. A third group explored a reduced MATLAB model of the FKC, MFK and governor system and its response to actual load data and given frequency series, and also on modifying the FKC to simultaneously constrain the time deviation while keeping frequency differences small.

Due to the complexity of the problem, it was not possible to satisfactorily resolve the problem during the week of the workshop. However, in this document we present the work that was completed during the study group, and some progress made since this time.

Participants in the workshop included Nabil Adam, from Transpower; facilitators Gerrard Liddell, Richard Brown, and Stephanie Hittmeyer; Geoffrey Pritchard, Dimetre Triadis, Ronald Begg, Mahbubeh Habibian, and 
Agate Ponder-Sutton.

\section{Modelling whole-network frequency regulation}

The power grid is a primarily a network of synchronous machines, all synchronised to the same frequency by direct electrical connection. New Zealand effectively has two major power grids, the North and the South Island. There is no direct AC connection between the North and South Islands, so they are not directly synchronised to each other. Instead the frequency synchronisation is achieved by control systems. The two islands are connected by a HVDC link, that allows power to be transmitted from one island to the other, to match up an imbalance between island loads and generation capacity.

Within an island, if the load drawn is higher than the prescribed amount of generation from the power stations, then the additional energy is taken from the kinetic energy stored in the various turbines, thus slowing them down and reducing the grid frequency. Conversely, if the load drawn is lower than the set point generation, then the system speeds up, and the grid frequency increases. To keep the frequency balanced, control systems are required which alter the set point values of the generators.

\subsection{Governor model-regulating governors}

In a standard model of a governor running in droop mode, the governor works to maintain the following relationship:

$$
\frac{1}{\mathrm{P}_{\max }}\left(\mathrm{P}-\mathrm{P}_{\mathrm{set}}\right)=-\frac{1}{\mathrm{R}}\left(\frac{\omega}{\omega_{\text {ref }}}-1\right)
$$

where $\mathrm{P}$ is the power generated by the generator, $\mathrm{P}_{\text {set }}$ is the set point generation, $\omega$ the system frequency, $\omega_{\text {ref }}$ is the reference frequency, and $R$ is a parameter 
called the droop, with a typical value of $0.04 . \mathrm{P}_{\max }$ is the maximum generation capacity of the unit. Under normal operating conditions, the grid frequency is very close to the reference $50 \mathrm{~Hz}$, and hence the power produced by the generator is very close to its set point. For a hydroelectric station, the governor can dynamically vary the amount of water flow to maintain this balance.

If the system frequency decreases, due to a higher load than set point on average across the system, then the RHS of (1) becomes positive, and so P is increased, meeting some of the additional load. All regulating generators on the network behave the same way, and so they work cooperatively to increase generation, bringing the frequency back up.

Only hydroelectric power stations work in this way, thermal ones do not, they simply produce their set point generation as long as the frequency remains within a deadband. For the purposes of this paper, frequency is assumed to always remain inside this deadband.

In the New Zealand system, the dispatch (set point) values are set every five minutes, and for our model, we treat the instantaneous generation every five minutes as dispatch for the following five minutes.

\section{$2.2 \quad$ Island model}

An island comprises many generators, all connected together and running at the same frequency. As described in the previous section, at equilibrium, for each thermal unit

$$
P_{j}=P_{s e t, j},
$$

and for each hydro unit

$$
P_{i}=P_{\text {set }, i}-\frac{P_{\max , i}}{R}\left(\frac{\omega}{\omega_{\text {ref }}}-1\right) .
$$

We assume that all generators have the same droop setting $R$ (as is standard practice). This means each generator participates proportionally in frequency 
regulation. Let the proportion of maximum generation capacity from hydro units be $\alpha$, that is,

$$
P_{\max }=\sum_{i} P_{\max , i}+\sum_{j} P_{\max , j}=\frac{1}{\alpha} \sum_{i} P_{\max , i} .
$$

Summing these, at equilibrium

$$
\begin{aligned}
P & =P_{\text {set }}-\left(\frac{\omega}{\omega_{\text {ref }}}-1\right) \sum_{i} \frac{P_{\text {max }, i}}{R} \\
& =P_{\text {set }}-\frac{\alpha P_{\max }}{R}\left(\frac{\omega}{\omega_{\text {ref }}}-1\right) .
\end{aligned}
$$

Finally, we solve for the steady state frequency

$$
\omega^{*}(P)=\omega_{\text {ref }}-\frac{\omega_{\text {ref }} R}{\alpha} \frac{P-P_{\text {set }}}{P_{\max }} .
$$

A simple linear model of the collective governor response for an island is

$$
\frac{d \omega}{d t}=-\frac{\omega-\omega^{*}(P)}{\tau_{G}}
$$

where the time constant $\tau_{\mathrm{G}}$ is of the order of five seconds.

This model does not explicitly capture the inertia of the generator systems, and the energy balance between generation, potential energy, and load. However, this first order linear system is a first approximation to these effects.

\subsection{MFK: island-level frequency control}

To control the frequency within an island, an MFK (multi frequency keeping) controller is used. This controller is allowed to make adjustments to the collective set point of the island, sharing it among a number of participating 
stations in a particular time period. It makes these adjustments in response to measurements of the error in system frequency. So $\mathrm{P}_{\text {set }}$ is decomposed as

$$
\mathrm{P}_{\mathrm{set}}=\mathrm{P}_{\text {dispatch }}-\mathrm{P}_{\mathrm{MFK}}
$$

However, there are certain limits between which this is allowed to act: $\mathrm{P}_{\mathrm{MFK}}$ is bounded by $\pm 20 \mathrm{MW}$ in the North Island and $\pm 10 \mathrm{MW}$ in the South Island. Additionally, there is a ramp rate limit of 8 and $4 \mathrm{MW} /$ second.

The controller is a PI (proportional/integral) controller, meaning it takes the form

$$
\mathrm{P}_{\mathrm{MFK}}=-\mathrm{K}_{\mathrm{G}} \Delta \omega-\mathrm{K}_{\mathrm{I}} \int_{0}^{\mathrm{t}} \Delta \omega \mathrm{ds}
$$

where $\Delta \omega=\omega-\omega_{\text {ref }}$, but subject to the additional constraints of maximum ramp rate and limiting.

Differentiating, and ignoring for the moment the ramp rate limit, we obtain a differential equation for $\mathrm{P}_{\text {MFK }}$ :

$$
\frac{d P_{M F K}}{d t}=-K_{G} \frac{d \omega}{d t}-K_{I} \Delta \omega .
$$

The expression for $\frac{d \omega}{d t}$ can be taken from the right hand side of (4).

Defining the new variables

$$
v=w-\omega_{\text {ref }}, \quad m=P_{\text {MFK }}, \quad u=P-P_{\text {dispatch }},
$$

and constant

$$
\sigma=\frac{\omega_{\text {ref }} R}{\alpha P_{\max }}
$$

we get the following coupled system

$$
\begin{aligned}
v^{\prime} & =\frac{1}{\tau_{G}}(-v+\sigma m+\sigma u) \\
m^{\prime} & = \begin{cases}-r a m p_{\max }, & \text { for }-K_{G} v^{\prime}-K_{I} v<- \text { ramp }_{\max }, \\
\operatorname{ramp}_{\max }, & \text { for }-K_{G} v^{\prime}-K_{I} v>\operatorname{ramp}_{\max }, \\
-K_{G} v^{\prime}-K_{I} v, & \text { otherwise. }\end{cases}
\end{aligned}
$$


If $\mathrm{m}^{\prime}$ stays below the maximum ramp rate, for fixed $\mathfrak{u}$, then the system has a single equilibrium at $v=0, \mathfrak{m}=\mathfrak{u}$. So the effect of the MFK controller is to compensate for the discrepancy between the load and the dispatch value, driving the frequency error back down to zero.

The time error is computed by integrating the frequency error as $T_{e}(t)=$ $\int_{0}^{t} \omega-\omega_{\text {ref }} d \tau=\int_{0}^{t} \nu d \tau$.

\subsubsection{Some simple numerical experiments}

We performed some simple numerical tests of the MFK model to observe the frequency and time error characteristics under step changes of load (with respect to dispatch) with the hope of identifying system characteristics that cause an accumulation of time error.

Figure 1 demonstrates some of the notable characteristics of a single island model with MFK enabled. The first set of axes shows a stepped disturbance to the dispatch load, and then it is returned to normal in two smaller steps. The (shifted) frequency $v$ returns to equilibrium after each perturbation, and that the MFK signal $m$ shifts to offset the disturbance. Also, a net time error $T_{e}$ accumulates over the five minute interval, due to the ramp rate limit coming into effect. The reason for this error is that (5) is violated when the slow rate limit is in effect, and errors accumulate during this time.

For comparison, the same figure is plotted with the ramp rate limit disabled in Figure 2. The time error then resets to zero when the frequency disturbance returns to zero, consistent with (5). This simple observation shows that the nonlinearities in the MFK threshholding aparatus can cause a net time error accumulation. 
Figure 1: Single Island simulation with MFK. From top, $u$ is the deviation of generation from dispatch, $v$ the frequency deviation from $50 \mathrm{~Hz}, \mathrm{~m}$ the MFK signal, and $T_{e}$ the time error. We use approximate parameters for New Zealand's South Island, $\mathrm{P}_{\max }=3600 \mathrm{MW}, \alpha=0.97, \tau_{\mathrm{G}}=5 \mathrm{~s}, \mathrm{R}=0.04$, $\mathrm{K}_{\mathrm{G}}=250 \mathrm{MW} \mathrm{Hz}^{-1}, \mathrm{~K}_{\mathrm{I}}=5000 \mathrm{MW} \mathrm{s}^{-1}$

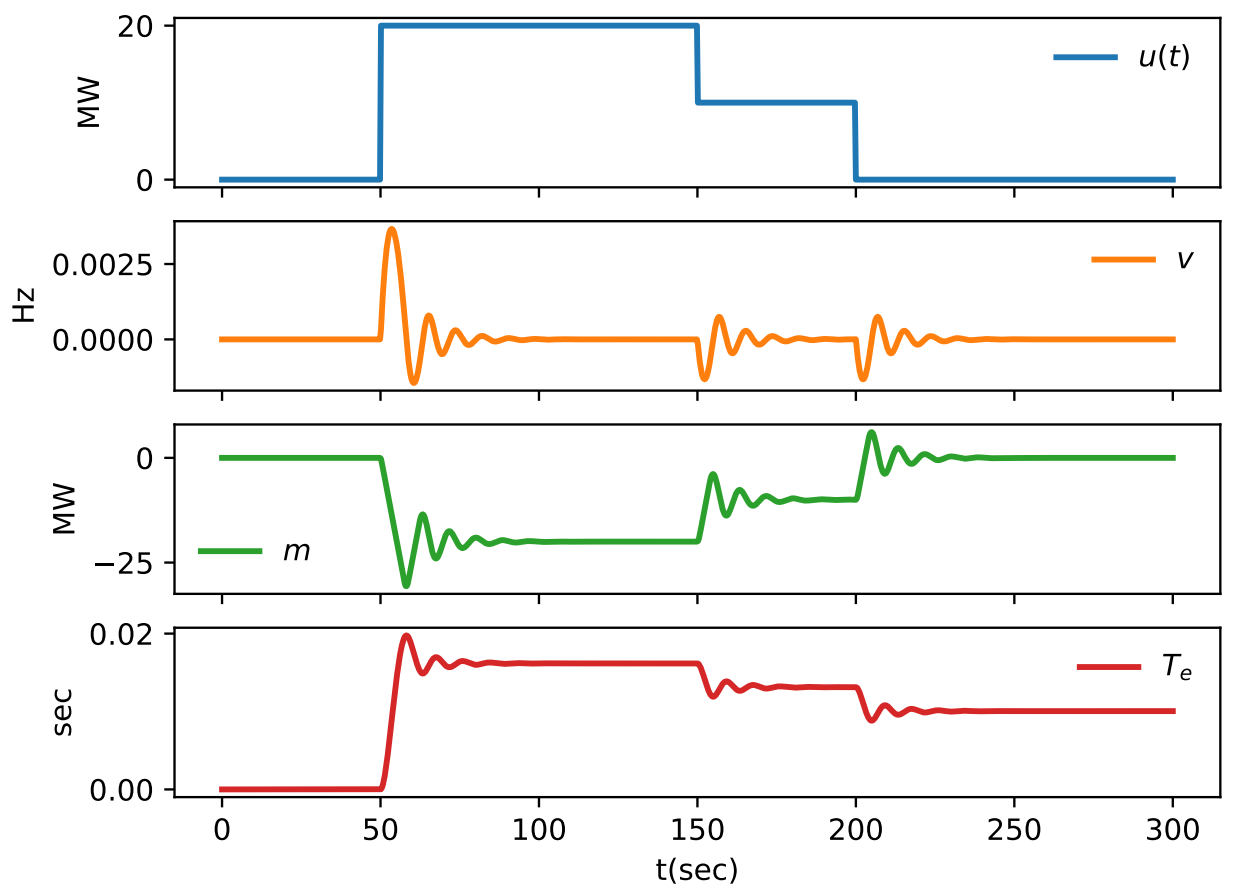


Figure 2: Single Island simulation with MFK, with ramp limit disabled. Note how the time error (bottom axes) resets to zero when the disturbance $\mathfrak{u}(\boldsymbol{t})$ returns to zero. We use approximate parameters for New Zealand's South Island, $\mathrm{P}_{\max }=3600 \mathrm{MW}, \alpha=0.97, \tau_{\mathrm{G}}=5 \mathrm{~s}, \mathrm{R}=0.04, \mathrm{~K}_{\mathrm{G}}=250 \mathrm{MW} \mathrm{Hz}^{-1}$, $\mathrm{K}_{\mathrm{I}}=5000 \mathrm{MW} \mathrm{s}^{-1}$

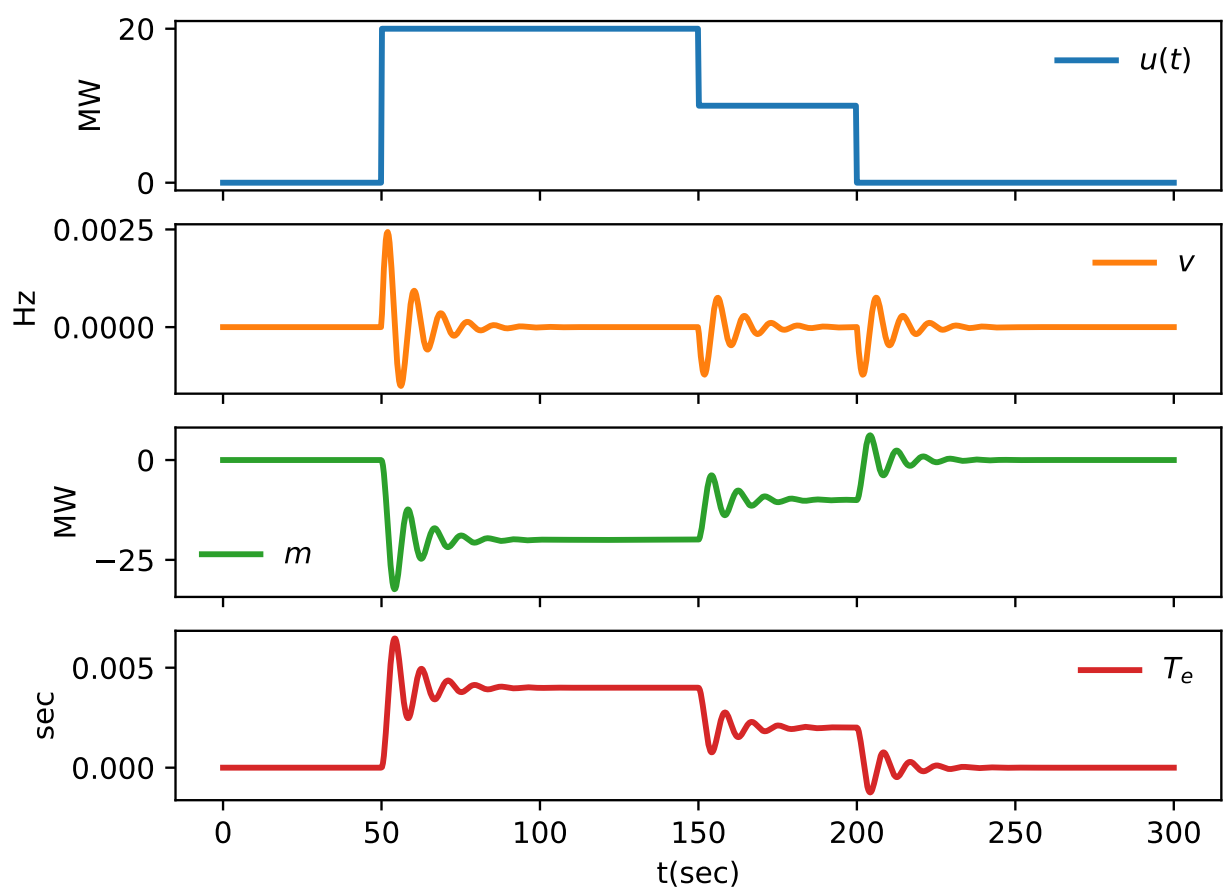




\subsection{FKC: interisland frequency synchronisation}

It is desirable that New Zealand has an electrical network that is synchronised across the whole country, rather than just in two islands. The connection between the islands is a High Voltage Direct Current (HVDC) link, which does not provide synchronous coupling between the networks. So instead, some kind of control strategy is required to synchronise the two systems.

Referring to (3), the quantities that influence system frequency are $P_{\text {set }}$ and $P$. So changes to the power station set points, $P_{\text {set }}$, or changes in the load or actual power generated, $\mathrm{P}$, influence the system frequency. The former is the kind of adjustment that MFK makes, and acts on a slow time scale, owing to the time taken to distribute MFK control signals to participating stations around the country. Therefore this mechanism is not a good candidate for network synchronisation. However, the latter can be modified by perturbing the amount of power being transmitted across the HVDC link between the islands, effectively lowering $\mathrm{P}$ for one island while raising $\mathrm{P}$ by the same amount for the other.

For example, suppose that $\mathrm{P}$ for South Island was $100 \mathrm{MW}$ higher than its $\mathrm{P}_{\text {set }}$. According to (3) and (4), this would result in a decrease in South Island frequency. However, if the overall HVDC current was adjusted by $+100 \mathrm{MW}$ in the North-South direction, then this would offset the difference, and the South Island target frequency $w^{*}$ would be correct. Of course this would upset the balance in the North Island, but the advantage of this mechanism is that power transmitted across the HVDC link can be adjusted almost instantaneously.

Because of the opposite directions of North Island / South Island frequency changes in response to the HVDC power perturbation, it is not possible for these perturbations to simultaneously regulate frequency of both Islands to the reference value. Rather, it either drives the frequencies closer together, or further apart. Hence the role of this system is to synchronise the frequencies of the two Islands, while the respective MFK controllers regulate the frequency itself. The control system that controls these HVDC perturbations with respect 
to capability is called the Frequency Keeping Control system (FKC).

In standard operation, there is a dispatch level of power transmission across the HVDC link, which is matched to the expected loads in the islands and the dispatch generation levels of the governors. The error signal that drives the controller is the frequency difference between the islands.

To incorporate FKC into our model, we model each island system using the whole-island model of Subsection 2.2. However, in this mode of operation, there is only one MFK system operating, and this is tied to either the South or the North Island frequency error. Around two-thirds of the MFK participants are in the South Island, due to the higher proportion of hydroelectric stations there, and the other third in the North Island.

Let the FKC current from North to South be F. Then we extend our model (7) as follows ( $\mathrm{N}$ subscripts for North Island, $\mathrm{S}$ subscripts for South Island):

$$
\begin{array}{lll}
v_{N}=\omega_{N}-\omega_{\text {ref }}, & v_{S}=\omega_{S}-\omega_{\text {ref }}, & m=P_{M F K}, \\
u_{N}=P_{N}-P_{\text {dispatch, } N}+F, & u_{S}=P_{S}-P_{\text {dispatch, },}-F . &
\end{array}
$$

The FKC provides additional power transmission proportional to the instantaneous frequency difference between the two islands. That is,

$$
\mathrm{F}=\gamma\left(\omega_{\mathrm{N}}-\omega_{\mathrm{S}}\right)=\gamma\left(v_{\mathrm{N}}-v_{\mathrm{S}}\right),
$$

where $\gamma$ is a constant. We take $\gamma=40,000 \mathrm{MW} / \mathrm{Hz}$ by manual tuning of the model. The differential equations for North and South island frequency (7) are adjusted as follows to reflect the MFK split

$$
\begin{aligned}
& v_{N}^{\prime}=\frac{1}{\tau_{G}}\left(-v_{N}+\frac{1}{3} \sigma_{N} m+\sigma_{N} u_{N}\right), \\
& v_{S}^{\prime}=\frac{1}{\tau_{G}}\left(-v_{S}+\frac{2}{3} \sigma_{S} m+\sigma_{S} u_{S}\right),
\end{aligned}
$$

where

$$
\sigma_{N}=\frac{\omega_{\text {ref }} R}{\alpha_{N} P_{\max , N}} \quad \text { and } \quad \sigma_{S}=\frac{\omega_{\text {ref }} R}{\alpha_{S} P_{\max , S}} i
$$


and $P_{\max , N}, P_{\max , S}, \alpha_{N}$, and $\alpha_{S}$ are defined as in Subsection 2.2. The MFK equation remains the same, but is referred to as either $v_{N}$ or $v_{S}$.

\subsubsection{Some numerical experiments}

In order to check the correct functioning of the FKC system, we ran some numerical experiments with a load with occasional sudden step and constant oscillation. Figure 3 shows that despite the obviously different load patterns, the FKC successfully locks the frequencies and time errors of the two islands together.

Figure 4 illustrates what happens if the FKC is insufficiently strong to synchronise the frequencies $(\gamma=10)$. The frequencies are not synchronised, and in this particular case MFK controls the frequency of the island it is locked to (South). Finally, Figure 5 illustrates the effect of switching off the MFK system, with FKC enabled. The frequency, and in particular the time error, vary significantly more with MFK off, and so in the synchronised system the MFK still plays an important role.

A variety of combinations of parameters were tested. However, we were unable to discover anything in the presented model that was able to give rise to the significant rapid time error creep that was observed in the real system, only the ramp rate limit in the MFK controller was able to give rise to a sustained time error.

\section{Conclusions, and future work}

Unfortunately we were unable to discover a potential source of the sudden rapid time error creep through this modelling process, but we hope that the model produced may be of some utility in understanding properties of the system or be of educational value. For it to be of more practical use, parameters will need to be tuned so that if real world data on load 
Figure 3: FKC and MFK both enabled. North Island traces in blue, South Island in red

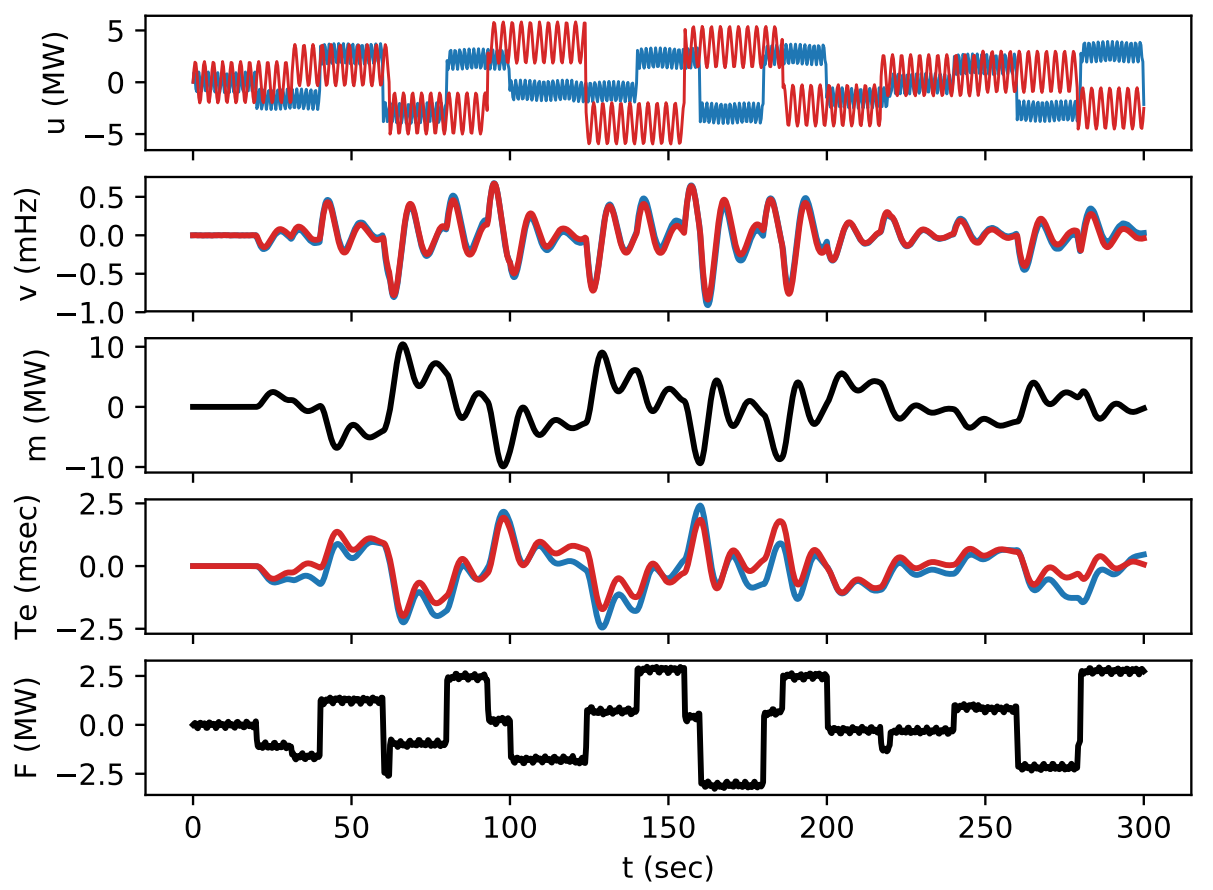

and dispatch is incorporated, then the system will mimic the actual power network. Presently sensible estimates of parameter values have been used, with data taken from Transpower documentation, private communications, and publicly available information on the New Zealand generation system.

A potential source of time error creep that has not been investigated in these models is the delay in propagation between the MFK signal being calculated, and the participating governors being actuated. The disparity between the islands may also contribute in some manner to this. Mathematically, this is 
Figure 4: FKC disabled, and MFK enabled. North Island traces in blue, South Island in red

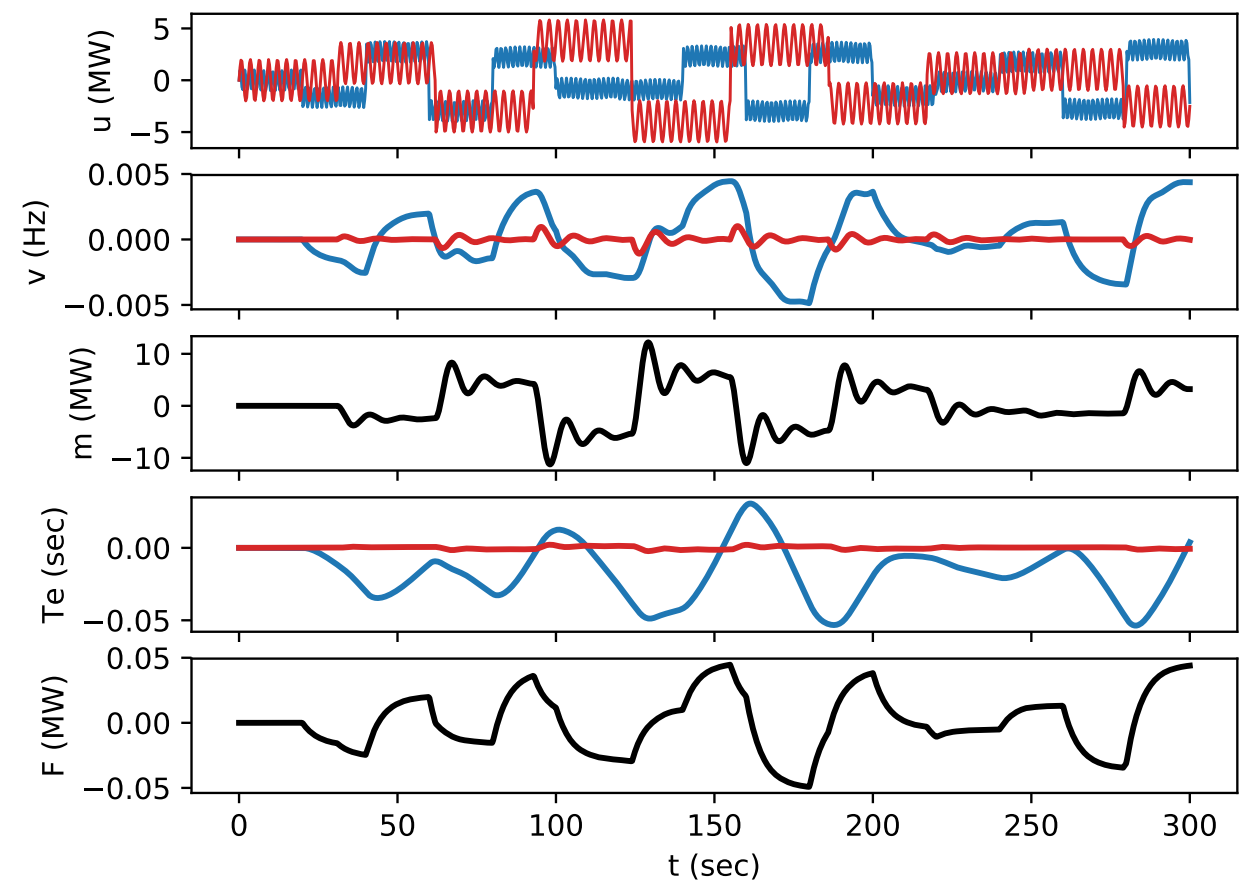

the domain of delay differential equations. Additionally, the effects of the MFK nonlinearities have not been comprehensively examined.

Finally, we have only presented in detail one aspect of the problem that was studied during the MINZ week. The other approaches involving statistical analysis of the time error data, and study of Transpower's Simulink model of the power generation system are still in progress and will be published at a later date. 
Figure 5: FKC enabled and MFK disabled. North Island traces in blue, South Island in red
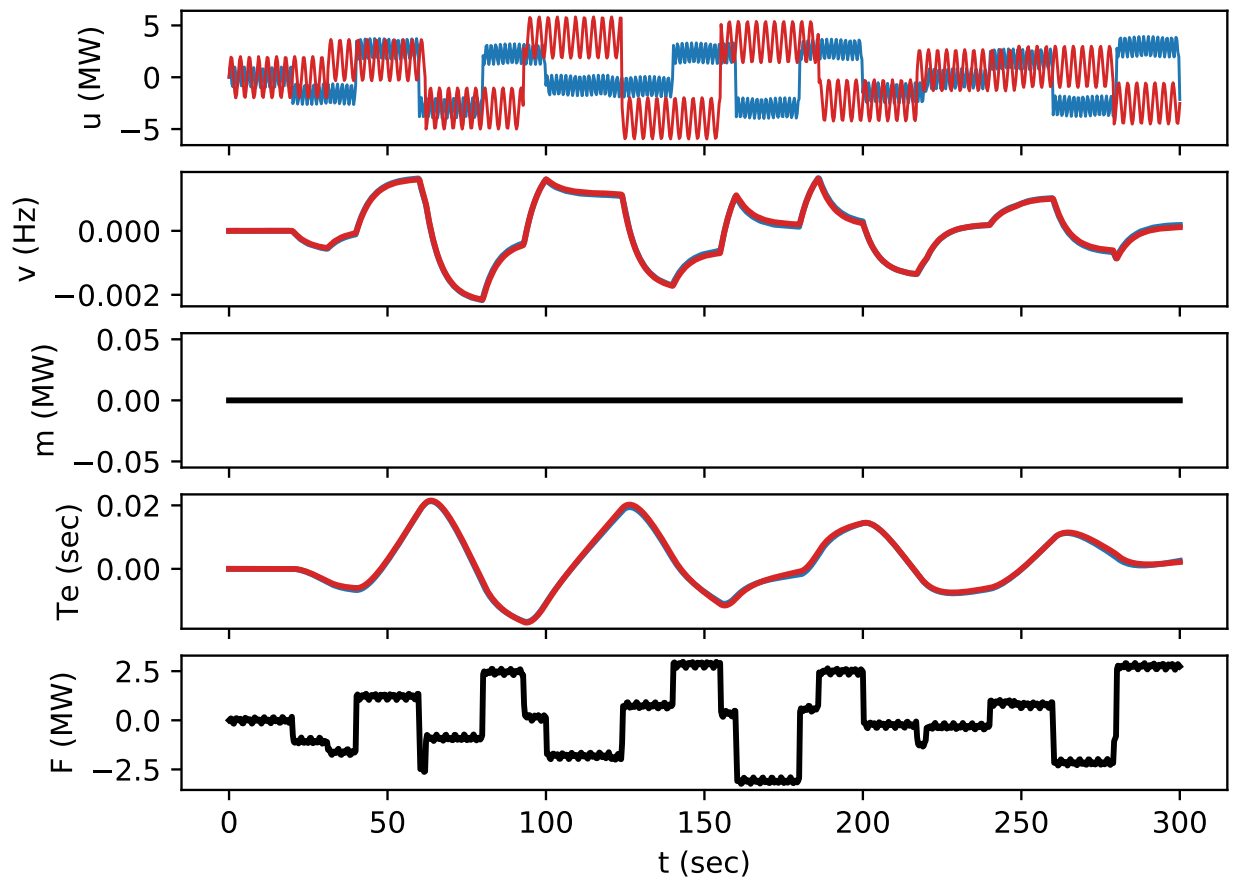

\section{Author addresses}

1. Richard G. Brown, Institute of Fundamental Sciences, Massey University (Palmerston North) mailto:r.g.brown@massey . ac.nz orcid:0000-0001-6926-4184

2. Gerrard Liddell, Department of Mathematics, University of Otago mailto:gliddell@maths.otago.ac.nz 
3. Nabil Adam, Transpower

mailto:Nabil. Adam@transpower.co.nz 